\title{
Periodontal Disease and Bisphosphonates Induce Osteonecrosis of the Jaws in the Rat
}

\author{
Tara L Aghaloo ${ }^{1}$, Ben Kang ${ }^{1}$, Eric C Sung ${ }^{2}$, Michael Shoff ${ }^{1}$, Matthew Ronconi ${ }^{1}$, Jack E \\ Gotcher $^{3}$, Olga Bezouglaia ${ }^{1}$, Sarah M Dry ${ }^{4}$, and Sotirios Tetradis ${ }^{1,5}$ \\ ${ }^{1}$ Division of Diagnostic and Surgical Sciences, UCLA School of Dentistry, Los Angeles, CA, USA \\ ${ }^{2}$ Division of Advanced Prosthodontics, Biomaterials, and Hospital Dentistry, UCLA School of \\ Dentistry, Los Angeles, CA, USA \\ ${ }^{3}$ Department of Oral and Maxillofacial Surgery, University of Tennessee Knoxville, Knoxville, TN, \\ USA \\ ${ }^{4}$ Department of Pathology and Laboratory Medicine, David Geffen School of Medicine at UCLA, \\ Los Angeles, CA, USA \\ ${ }^{5}$ Molecular Biology Institute, UCLA, Los Angeles, CA, USA
}

\begin{abstract}
Bisphosphonates (BPs) are medications used commonly to treat primary and metastatic bone cancer, as well as osteoporosis. Although BPs improve bone mineral density, reduce fracture risk, and reduce hypercalcemia of malignancy, some patients develop BP-related osteonecrosis of the jaws (BRONJ). This devastating complication is defined as clinically exposed bone in the maxillofacial region for more than 8 weeks. Despite an increasing number of BRONJ cases since first reported, the disease pathophysiology remains largely unknown. Since published studies suggest a significant role for dental disease in the pathophysiology of BRONJ, we developed a BRONJ animal model where aggressive periodontal disease is induced by ligature placement around the crown of the right maxillary first molar in the presence of vehicle (veh) or zoledronic acid (ZA), a potent BP. Ligature placement induced significant alveolar bone loss, which was attenuated by ZA treatment. Osteonecrosis was observed associated with ligature-induced periodontitis in the ZA-treated group. This was seen as sequestration and extensive periosteal alveolar bone formation on micro-computed tomography $(\mu \mathrm{CT})$ in the ligated site of BP-treated animals. Histologic examination confirmed these findings, seen as necrotic bone with diffuse loss of osteocytes and empty lacunae, rimming of the necrotic bone by squamous epithelium and inflammation, and exposure to the oral cavity. Importantly, the rat lesions were strikingly similar to those of BRONJ patients. Our data suggest that dental disease and potent BP therapy are sufficient for BRONJ development in the rat.
\end{abstract}

\section{Keywords}

OSTEONECROSIS OF THE JAWS; BISPHOSPHONATE; PERIODONTITIS; RAT MODEL; BRONJ; ONJ

\footnotetext{
(C) 2011 American Society for Bone and Mineral Research

Address correspondence to: Sotirios Tetradis, DDS, PhD, Diagnostic and Surgical Sciences, UCLA School of Dentistry, 10833 LeConte Avenue, CHS Room 53-068, Los Angeles, CA 90095-1668, USA. stetradis@ dentistry.ucla.edu.

Disclosures All the authors state that they have no conflicts of interest.
} 


\section{Introduction}

Bisphosphonate-related osteonecrosis of the jaws (BRONJ) is defined clinically as an area of exposed bone in the maxillofacial region that did not heal within 8 weeks of identification by a health care provider in a patient who was receiving or had been exposed to a bisphosphonate and had not had radiation therapy to the craniofacial region. ${ }^{(1,2)}$ The clinical presentation is similar to osteomyelitis (OM) or osteoradionecrosis (ORN) of the jaws, the former caused by an infection in the bone and the latter by high doses of localized radiation therapy. ${ }^{(3-5)}$ However, BRONJ patients have no history of radiation therapy and may have a secondary bacterial infection, and classic therapies for OM and ORN are usually ineffective. ${ }^{(3,6,7)}$ Although studies do not identify a direct causal relationship between BP use and osteonecrosis of the jaws $(\mathrm{ONJ}),{ }^{(8,9)}$ the only consistent variable for many ONJ cases is BP therapy, most commonly through intravenous administration. From these data, the primary involvement of BPs in the pathophysiology of ONJ is strongly suggested. ${ }^{(3,6,7,10,11)}$

Overall, the risk of BRONJ development for patients on intravenous BPs is estimated at between $1 \%$ and $11 \%,{ }^{(10-14)}$ and it increases after longer treatment time and in patients on zoledronic acid (ZA). ${ }^{(6)}$ BRONJ may be underreported because much of the published clinical literature consists of retrospective chart reviews and because many patients do not have documented routine dental examination during treatment. ${ }^{(14,15)}$ BRONJ in patients taking oral BPs for osteoporosis is rare, estimated at $0.001 \%$ to $0.1 \% .^{(1,16,17)} \mathrm{A}$ recent Kaiser Permanente study demonstrates an increased number of BRONJ cases possibly owing to chronic oral BP use and thorough screening. ${ }^{(17)}$

In the great majority of cases, BRONJ occurs after extraction of teeth deemed unrestorable owing to the severity of dental disease or around teeth with active periodontal or periapical disease. ${ }^{(2,3,15,18,19)}$ In a series of 152 BRONJ cases, for 85 patients (56\%), the initiating event for ONJ was either extraction owing to periodontitis, extraction owing to failing root canal, uncontrolled periodontitis, periodontal surgery, or apicoectomy. An additional 25 patients $(16.4 \%)$ received tooth extraction owing to caries; it would be safe to assume that for most of these 25 patients, tooth extraction was required for deep caries that extended to the pulp with associated pulpal necrosis and periapical inflammation. ${ }^{(20)}$ Thus, although not always reported as the initiating factor, inflammation of the periodontal tissues is present in the majority of BRONJ cases. Moreover, oral preventive measures decrease BRONJ incidence, ${ }^{(21,22)}$ further emphasizing the importance of dental disease in BRONJ pathophysiology.

To investigate the significance of dental disease in the development of BRONJ, we have developed a clinically relevant animal model. We induced aggressive periodontal inflammation in rats treated with vehicle or ZA, a potent BP. We observed alveolar bone necrosis with bone sequestration and/or periosteal bone reaction. Importantly, the appearance of the osteonecrotic bone in the rat closely resembled BRONJ in patients.

\section{Materials and Methods}

\section{Animal care and ligature placement}

All animals and surgical procedures were handled in accordance with guidelines of the Chancellor's Animal Research Committee of the Office for Protection of Research Subjects at the University of California, Los Angeles. Three-month-old male Sprague-Dawley rats (Charles River Laboratories, Wilmington, MA, USA) were housed in light- and temperature-controlled facilities and given food and water ad libitum. Animals received intraperitoneal injections of vehicle (sterile saline) or $66 \mu \mathrm{g} / \mathrm{kg}$ of ZA three times per week 
for 3 weeks prior to intraoral procedures. This dose corresponds to the 4-mg/60-kg ZA dose administered monthly to cancer patients for bone disease control. ${ }^{(23,24)}$ Nineteen animals in each of the vehicle- (veh) or ZA-treated groups were used.

For all our studies described herein, we adapted the well-characterized ligature model by placing a sterile wire ligature around the crown of the right first maxillary molar (Fig. 1A). Rats were anesthetized with isoflurane, and a sterile 28-gauge wire ligature was placed around the cervical portion of the right first molar. ${ }^{(25)}$ Animals continued to receive vehicle or ZA injections for 12 more weeks after ligature placement. Animals were monitored weekly to ensure presence of the ligature, and the ligature was adjusted if necessary. All animals were given a regular diet throughout the duration of the experiment.

Weight of animals was measured three times per week. At the end of the experiment, animals were euthanized, blood was collected via cardiac puncture, and serum biomarkers including $\mathrm{Na}, \mathrm{K}, \mathrm{Cl}, \mathrm{CO}_{2}$, blood urea nitrogen (BUN), creatinine, $\mathrm{Ca}, \mathrm{Mg}$, phosphorus, albumin, and alkaline phosphatase were measured. No significant differences in weight or any of the serum markers between vehicle- and ZA-treated animals was detected (data not shown). Whole maxillas were removed, placed in $10 \%$ formalin for 48 hours, and stored in $70 \%$ ethanol.

\section{Micro-computed tomographic $(\mu \mathrm{CT})$ scanning}

Bones were imaged by $\mu \mathrm{CT}$ scanning ( $\mu \mathrm{CT} 40$; Scanco Medical AG, Basserdorf, Switzerland) at $16-\mu \mathrm{m}$ resolution, and volumetric data were converted to DICOM format and imported in the Dolphin Imaging software (Chatsworth, CA, USA) to generate 3D and multiplanar reconstructed images. An oral and maxillofacial radiologist (ST), blinded to the specific animal treatment, evaluated the $\mu \mathrm{CT}$ images of all animals to identify and score bony changes and performed all linear measurements using Dolphin software tools.

To quantify the amount of bone loss induced by experimental periodontal disease (PD), the imaged volume was oriented with the nasal cavity floor parallel to the horizontal plane and the midpalatal suture parallel to the midsagittal plane. Then the volume was angled such that the long axis of the distal root of the first molar (D1) and the mesial root of the second molar (M2) were vertical to the horizontal plane. Then the distance between the cementoenamel junction (CEJ) and the alveolar bone crest (ABC) was measured at the center of D1 and M2 (Fig. $1 B$ ). To quantitatively assess changes in the width of the buccal alveolar outline on axial slices, the imaged volume was oriented such that the floor of the nasal cavity was parallel to the horizontal plane and the midpalatal suture was parallel to the midsagittal plane. Then the shortest distance from the buccal surface of the root to the buccal outline of the alveolar ridge was measured for the mesial and distal roots of the first and second molars at the level of the hard palate (Fig $5 A$ ).

\section{Histology and TUNEL staining}

Bones were decalcified in Cal-Ex II Fixative/Decalcifier (Fisher Scientific, Pittsburgh, PA, USA) for 4 to 6 days or in $14.5 \%$ EDTA (pH 7.2) for 4 weeks. Samples then were embedded in paraffin, and 5- $\mu \mathrm{m}$-thick coronal sections at the interproximal area between the first and second maxillary molars were made. Thus each section included a complete cross section through the entire maxilla, which allowed a side-by-side comparison of the bone, teeth, and soft tissues from the ligature (right) and nonligature (left) sites.

To quantify the area of osteonecrosis and periosteal thickness, hematoxylin and eosin (H\&E)-stained slides were digitally scanned using the Aperio XT automated slide scanner and the Aperio ImageScope Version 10 software (Aperio Technologies, Inc., Vista, CA, USA). One author (SD) marked the area(s) of osteonecrosis, defined as loss of more than 
five contiguous osteocytes with confluent areas of empty lacunae, and the total area was calculated by the ImageScope software. The ruler tool in ImageScope was used to measure the greatest area of buccal periosteal thickness on both the ligature and nonligature sides. Numbers of empty and total osteocytic lacunae were counted manually on the digital wholeslide image over a $1-\mathrm{mm}$-long and $0.25-\mathrm{mm}$-wide area of bone (length and width measured with the ImageScope ruler tool) at the buccal alveolus adjacent to the D1 root.

Terminal deoxynucleotidyl transferase-mediated dUTP nick-end labeling (TUNEL) staining using the DeadEnd Colorimetric TUNEL System Kit (Promega, San Luis Obispo, CA, USA) was performed on the section adjacent to that used for the H\&E stain. For TUNEL quantification, $\mathrm{TUNEL}^{+}$osteocytes and total osteocytes were counted manually within 1 $\mathrm{mm}$ adjacent to osteonecrotic foci or (if osteonecrosis was not present) within a $1 \mathrm{~mm}$ along the buccal alveolar bone.

Using a standard brightfield microscope, areas of mucosa overlying the alveolar crest with the greatest inflammation were identified. The high-power $(x 40)$ field with the greatest numbers of inflammatory cells was selected, and the numbers of polymorphonuclear cells and lymphocytes were counted manually.

All histology and digital imaging were performed at the Translational Pathology Core Laboratory (TPCL) at UCLA.

\section{Radiographic evaluation of patient records}

Approval by the Office for Protection of Research Subjects at the University of California Los Angeles was obtained to review the radiographic records of 17 patients with a diagnosis of BRONJ. Seventeen patients were imaged at the Oral and Maxillofacial Radiology Clinic at the UCLA School of Dentistry using a high-resolution cone-beam computed tomographic (CBCT) 3D Accuitomo scanner (J Morita, Japan). BRONJ and unaffected sites were reviewed, and the cortical thicknesses of the buccal or lingual cortex of the affected alveolar ridge and of the corresponding site of healthy alveolar ridge were measured in axial, coronal, or sagittal reconstructed sections. Then the cortical thickness of the alveolar ridge at the BRONJ site was expressed as a percent thickness of the healthy site.

\section{Statistics}

Data among groups were analyzed using one-way analysis of variance (ANOVA) and the Student-Newman-Keuls post hoc test. Data between groups were analyzed using Student's $t$ test.

\section{Results}

To ensure that ligature placement was effective in inducing experimental periodontal disease (PD), dissected maxillas were imaged by $\mu \mathrm{CT}$ as described earlier. The distance from the CEJ to the AC was measured at the distal buccal root of the first maxillary molar (D1) and the mesial buccal root of the second maxillary molar (M2) in both the ligature and nonligature sites (right and left, respectively; Fig. $1 B$ ). Increase in the CEJ-AC distance represents bone loss. Ligature placement caused a significant increase in the CEJ-AC distance for D1 and M2 in both vehicle- and ZA-treated animals (Fig. 1C, D). Interestingly, though, vehicle-treated animals experienced augmented periodontal bone loss, as reflected by the significantly increased CEJ-AC distance compared with the ZA-treated animals (Fig. $1 C, D)$.

Then multiplanar and 3D re-formatted $\mu \mathrm{CT}$ images from vehicle- and ZA-treated animals were reviewed for evaluation of radiographic findings indicative of osteonecrosis. 
Representative 3D re-formatted images of nonligated and ligated maxillas from vehicle- or ZA-treated animals are depicted in Fig. 2. Maxillas in the nonligated site from both vehicle(Fig. $2 A$ ) or ZA-treated animals (not shown) demonstrate a normal alveolar crest that extends just inferior of the CEJ, covering most of the root, as well as the root furcation of the first and second molars (purple arrow, Fig. $2 A$ ). In the ligated site of vehicle-treated animals, loss of alveolar crest height with root exposure and furcation areas characteristic of periodontal bone destruction were seen (yellow arrow, Fig. 2B). In contrast, the alveolar bone of the ligated site of nine ZA-treated animals presented a different radiographic appearance. Some animals showed radiographic features of sequestrum (a necrotic piece of bone) seen as bony fragments with irregular borders that were separated from the remaining alveolar ridge (orange arrow, Fig. 2C). In other ZA-treated animals, substantial expansion of the buccal thickness of the alveolar ridge, radiographically consistent with extensive periosteal new bone formation, was observed (green arrow, Fig. 2D). Sequestra, periosteal bone formation, and alveolar ridge expansion are common radiographic findings in BRONJ patients. ${ }^{(26,27)}$

We compared the radiographic appearance of the alveolar ridge from the ZA-treated animals with that of BRONJ lesions from patients treated at the UCLA School of Dentistry Clinic. Both ZA-treated rats and BRONJ patients showed similar features of sequestra and alveolar bone expansion. Figure 3 presents $\mu \mathrm{CT}$ multiplanar views from a ZA-treated rat (from Fig. $2 C$ ) and corresponding multiplanar CT views from a patient. Both rat and patient images demonstrate an irregular radiopacity surrounded by an erosive radiolucent zone, consistent with a bony sequestrum that is discontinuous from the remaining alveolar bone; this is seen in sagittal, coronal, and axial slices (Fig. 3, arrows). Figure 4 presents $\mu \mathrm{CT}$ multiplanar views of a ZA-treated rat (from Fig. 2D) and corresponding multiplanar CT views from another BRONJ patient. Both rat and patient images demonstrate the appearance of substantial new bone formation at the periphery seen in sagittal, coronal, and axial slices (Fig. 4, arrows) that causes significant expansion of the alveolar ridge (compare affected with unaffected sides in Fig. 4 coronal and axial slices for the rat).

Because extensive periosteal bone formation was observed in the ligated sites of several ZAtreated animals, we quantitated the buccal bone thickness in the ligated versus nonligated sites in vehicle- versus ZA-treated animals (Fig. 5). The buccal width of the alveolar bone was measured at the mesial and distal buccal roots of the first (M1 and D1) and second (M2 and D2) maxillary molars (Fig. 5A). An increase in buccal width of the ligated site was observed in vehicle-treated animals and involved D1, M2, and D2 (Fig. 5B). Importantly, ZA-treated animals demonstrated a significantly higher increase in the buccal width in ligated versus nonligated sites compared with vehicle-treated animals that extended to a larger area involving M1, D1, M2, and D2 (Fig. 5B).

To investigate whether increased alveolar bone width is also seen in BRONJ patients, we measured the cortical thickness of the alveolar bone at BRONJ lesions and the same alveolar bone area of the noninvolved site on multiplanar CBCT images of 17 patients treated at the UCLA School of Dentistry Clinic (Fig. 5C). A twofold statistically significantly increased alveolar cortical thickness was observed at the BRONJ site compared with the non-BRONJ site (Fig. 5D).

To evaluate the histologic appearance of the alveolar bone and periodontal ligament at the nonligated and ligated sites of vehicle- versus ZA-treated animals, sections from the D1 to the M2 roots were examined following decalcification and H\&E staining (Fig. 6). Sections from the nonligated site of vehicle-treated animals (Fig. 6A, $A I$ ) or ZA-treated animals (not shown) showed viable lamellar bone surrounding nearly the entire tooth root length (black arrow). Sections from the ligated site of vehicle-treated maxillas (Fig. 6B, BI) demonstrated 
inflammation (green arrow) and marked bone loss/resorption; viable lamellar bone surrounded the lower $25 \%$ of the root (black arrow). In sections from the ligated sites of ZAtreated animals (Fig. 6C, CI), necrotic lamellar bone (yellow arrows), characterized by diffuse loss of osteocytes with confluent areas of empty lacunae ( $C 1$ inset, $D 1$ inset), was observed. In Fig. $6 C$ and $C 1$, the mucosal surface of this necrotic bone is not covered by oral mucosal epithelium and is exposed to the oral cavity (red arrow); deeper portions of the sequestrum are rimmed by squamous epithelium (blue arrow). In Fig. $6 D$ and $D 1$, substantial periosteal bone formation (double white arrow) is present adjacent to necrotic bone; unlike in Fig. $6 C$, the necrotic bone is not sequestered but is continuous with the remaining viable bone (black arrow). Inflammation continues to be seen in the ligated site with ZA treatment (green arrows, Fig. $6 C, C 1, D, D 1$ ).

This histologic appearance of necrotic bone with exposure to the oral cavity confirms osteonecrosis of the maxilla in ZA-treated rats with ligature-induced PD. To evaluate the histologic fidelity of our rat model for human BRONJ, we compared the appearance of the ZA-treated rat with osteonecrosis with a histologic specimen from a BRONJ patient (Fig. 7). Similar to our rat model (Fig $7 A, C$ ), sections from a human BRONJ patient (Fig. 7B, $D$ ) demonstrated osteonecrosis of lamellar bone with empty lacunae (yellow arrows) and rimming of necrotic bone by squamous epithelium (blue arrows) and inflammation (green arrows).

Table 1 summarizes the radiographic and histologic findings of the 19 vehicle- and $19 \mathrm{ZA}-$ treated animals that were included in our studies. Radiographically, 6 (32\%) ZA- and 1 (5\%) vehicle-treated animals presented irregular bone fragments detached from the surrounding alveolus, characteristic of sequestrum formation. Sequestrum volume was $1.404 \mathrm{~mm}^{3}( \pm$ $\left.0.520 \mathrm{~mm}^{3}\right)$. Additionally, $6(32 \%)$ ZA-treated animals displayed a greater than twofold increase in buccal cortical width compared with the nonligated site, suggestive of substantial periosteal bone formation. No vehicle-treated animal demonstrated such a dramatic increase in buccal alveolar bone thickness. Histologically, 4 (21\%) ZA-treated animals showed bone not covered by epithelium, indicating clinical bone exposure. Finally, 9 (47\%) ZA- and 1 $(5 \%)$ vehicle-treated animals revealed clear histologic presentation of bone necrosis with extensive empty osteocytic lacunae. The area of histologic bone necrosis was $0.303 \mathrm{~mm}^{2}( \pm$ $0.073 \mathrm{~mm}^{2}$ ).

To evaluate the area of bone necrosis in more detail, the percent empty over total osteocytic lacunae at the buccal alveolus adjacent to D1 root was determined (Fig. 8A). Normally, there is a rim of empty space between the osteocyte and the rim of the lacunae. Depending on the plane of section through the bone, scattered empty lacunae may be seen in viable bone. In contrast, osteonecrotic bone shows diffuse loss of osteocytes within a region. ${ }^{(28)}$ Importantly, no difference was observed between the ligated and nonligated sites of vehicletreated animals or the nonligated site of ZA-treated animals. However, the ligated site of ZA-treated animals showed a significant increase in the number of empty osteocytic lacunae (Fig. $8 A$ ).

Because of the $\mu \mathrm{CT}$-observed increased periosteal bone formation (Fig. $5 B$ ), we measured the thickness of the periosteum at the buccal aspect of the alveolar ridge (Fig. $8 B$ ). The ligated site of ZA-treated animals showed a significantly increased periosteal thickness, whereas no differences were observed in the other group. Gingival inflammation induced by the ligature was similar in vehicle- and ZA-treated animals, with increased numbers of polymorphonuclear neutrophils (PMNs) that were statistically significantly different compared with the nonligated site but not different between vehicle- and ZA-treated animals (Fig. 8C). Ligature also increased the number of lymphocytes in both vehicle- and ZA- 
treated animals (Fig. 8D). Although this did not reach statistical significance $(p=.0557)$, lymphocyte increase was higher in ZA-treated animals.

Finally, osteocytes adjacent to the osteonecrotic areas appeared pyknotic, with nuclei that were shrunken and more basophilic. We therefore performed TUNEL assays to investigate the presence of impending cell death via the apoptotic pathway (Fig. 9). Only isolated $\mathrm{TUNEL}^{+}$osteocytes were seen in sections from nonligated or ligated sites of vehicle-treated animals (Fig. 9A, B) or from the nonligated site of ZA-treated animals (Fig. 9C). In contrast, $\mathrm{TUNEL}^{+}$osteocytes were seen close to osteonecrotic regions in the ligated site of ZAtreated animals, including those areas with pyknotic-appearing nuclei on H\&E stain (Fig. $9 D$ ). Quantification of percent $\mathrm{TUNEL}^{+}$over total osteocytes revealed a statistically significant increase in the ligated site of ZA-treated animals (Fig. 9E).

\section{Discussion}

Clinically, BPs have advanced therapy for malignant diseases such as multiple myeloma and breast and prostate cancer by decreasing life-threatening skeletal-related complications, including hypercalcemia of malignancy, pathologic fractures, spinal cord compression, and severe bone pain. ${ }^{(29,30)}$ Long-term studies suggest that potent BPs such as ZA decrease tumor burden in animals ${ }^{(31)}$ and improve quality of life and survival in certain patient groups. ${ }^{(32)}$ BPs are also very effective in treating osteoporosis and Paget disease by increasing bone mineral density, decreasing fracture risk, and inhibiting bone resorption, respectively. ${ }^{(9,33,34)}$

Despite the great clinical benefits in cancer and osteoporotic patient management, BP use has been associated recently with BRONJ. ${ }^{(2,15)}$ To date, a major unanswered question is the occurrence of BP-related osteonecrosis only in the jaws, sparing the long bones and axial skeleton. The orofacial complex is a complicated system composed of teeth, oral mucosa, periodontal tissues, muscles, tongue, salivary glands, and alveolar bone. These tissues interact to perform unique functions that range from mastication and speech to swallowing and taste. The maxilla and mandible are the only bones covered by mucosa in close proximity to the external environment, ${ }^{(35)}$ where bacterial infections such as caries and periodontal disease occur commonly. ${ }^{(36,37)}$ Hypotheses that attempt to explain the specificity of BRONJ include altered bone remodeling, angiogenesis inhibition, constant microtrauma, and bacterial infection. ${ }^{(3,38)}$ However, the specific parameters that contribute to the increased jaw sensitivity to BPs remain unidentified.

A recent in vivo study in beagle dogs that received 3 years of alendronate demonstrated matrix necrosis of alveolar bone, where the necrotic areas were void of patent canaliculi. Additionally, dynamic histomorphometry revealed a significant decrease in intracortical bone turnover. ${ }^{(39)}$ Similar results in the same animal model were observed with other BPs at different time points. ${ }^{(40)}$ Importantly, these BP effects occurred in the absence of jaw trauma or inflammation.

The absence of animal models to evaluate parameters that affect BRONJ occurrence, progression, and severity has been a major obstacle in understanding BRONJ pathophysiology. Recently, Sonis and colleagues demonstrated delayed wound healing with mucosal ulceration and bony sequestration after maxillary tooth extraction in rats that received ZA and dexamethasone (dex) combination therapy. ${ }^{(41)} \mathrm{A}$ very similar model of osteonecrotic lesions developing in mice treated with ZA and dexamethasone after extraction of maxillary first molars also was reported. ${ }^{(28,42)}$ These latest reports are very significant because they allowed adaptation of a BRONJ animal model with clear advantages for future studies using the rich experimental tools and genetic advantages of 
mouse models. In a parallel study, Hokugo and colleagues illustrated the importance of vitamin D deficiency in ZA-induced pseudoepitheliomatous hyperplasia, bony sequestration, and delayed wound healing after maxillary tooth extraction. Treatment with ZA alone did not result in osteonecrotic lesions, emphasizing the requirement of vitamin D deficiency in this model. ${ }^{(43)}$ Studies of periodontal disease induced by a high-carbohydrate soft diet ${ }^{(44,45)}$ in rice rats treated with dichloromethylene diphosphonate $\left(\mathrm{Cl}_{2} \mathrm{MDP} ; 0.1\right.$ to $\left.10 \mathrm{mg} / \mathrm{kg} / \mathrm{d}\right)$ described decreased bone loss in the $\mathrm{Cl}_{2}$ MDP-treated animals. Surprisingly, animals treated with high $\mathrm{Cl}_{2} \mathrm{MDP}$ doses developed trabecular bone protruding into the oral cavity and inflammatory infiltrate with loss of osteocytes, ${ }^{(45)}$ similar to the histologic and radiographic findings of osteonecrotic lesions in our study.

Most BRONJ lesions in patients appear after extraction of teeth that cannot be restored owing to advanced dental disease or around teeth with periodontal or periapical infection. ${ }^{(2,3,15,18,19)}$ Furthermore, aggressive dental hygiene reduces the incidence of BRONJ in multiple myeloma ${ }^{(21)}$ and metastatic cancer ${ }^{(2)}$ patients. It would be expected that alveolar bone inflammation from dental disease increases local bone turnover and thus enhances incorporation of BPs in the inflammatory area. Indeed, increased uptake of technetium-99m-labeled diphosphonate, indicating increased bone turnover, in the presence of periapical or periodontal disease is well documented. ${ }^{(46-50)}$

This apparent close association of active dental disease with human BRONJ prompted us to explore the necessary and sufficient requirement of aggressive PD and BP treatment in BRONJ development in a rat animal model. In this model, aggressive PD was induced with a sterile ligature around the maxillary first molar. Consistent with previous studies, ${ }^{(51)}$ significant alveolar bone loss occurred in the vehicle-treated animals, where the ligature was used. Also as expected, ZA treatment attenuated ligature-induced alveolar bone loss. ${ }^{(52)}$

Importantly, $\mu \mathrm{CT}$ analysis revealed significant changes in alveolar bone morphology in ZAtreated animals in the presence of PD. Sequestration or extensive periosteal bone formation, classic features of BRONJ in patients, ${ }^{(26,27)}$ were present in $47 \%$ of the PD-affected ZAtreated maxillas. Specifically, cortical bone thickness at the PD site of ZA-treated animals was significantly increased over the PD site of vehicle-treated rats. This increase mimicked BRONJ patient findings that demonstrated a significant increase in periosteal bone formation in the area of BRONJ compared with the unaffected contralateral site.

Histologically, the thickness of the periosteum lining the buccal surface of the alveolar ridge was significantly increased in the ligated site of ZA-treated animals, paralleling the radiographic findings of increased bone thickness in the same site. This effect does not appear to be a direct effect of BPs because the nonligated site illustrated normal bone and periosteal thickness but rather a combination of BP with experimental PD. In vivo BP effects on periosteum have been diverse. Alendronate and risedronate do not alter mineral apposition rate (MAR) at the periosteal rib surface of beagle dogs ${ }^{(53)}$ or mid-diaphysis of loaded or unloaded ulnas in ovariectomized rats. ${ }^{(54)}$ On the other hand, alendronate significantly increases MAR in femoral diaphysis periosteum of 1-week-old rats, ${ }^{(55)}$ whereas risedronate and alendronate suppress periosteal osteoblast activity in the tibias of 6month-old female rats. These variable findings may reflect species, age, or bone-site different responses of periosteum to BPs.

Histologic examination confirmed the presence of necrotic bone with diffuse loss of osteocytes and empty lacunae, diagnostic of osteonecrosis and the hallmark of BRONJ histopathology in humans. Apoptotic osteocytes, confirmed with TUNEL staining, were observed in the proximity of empty lacunae, indicating incipient osteocyte loss and expansion of the osteonecrotic areas. Histologically, the similarities between rat and patient were striking, both demonstrating necrotic bone with empty lacunae rimmed by squamous 
epithelium and inflammation. These findings were observed in 9 of 19 BP-treated animals with the induction of PD (47\%).

Interestingly, 1 of 19 vehicle-treated animals (5.2\%) developed an osteonecrotic lesion in the area of experimental PD. This finding was unexpected, and the reason for development of osteonecrosis in the absence of BP treatment is not clear at the current time. Common methods to induce experimental PD include lipopolysaccharide (LPS) injection in the interproximal gingivae of the maxillary molar teeth or ligature placement around the maxillary first molar crown. ${ }^{(25,56)}$ LPS injections create chronic inflammation with inflammatory cell infiltrate, increased inflammatory cytokines, and eventually, loss of alveolar bone. ${ }^{(25)}$ Alternatively, ligature placement leads to plaque accumulation and periodontal inflammation. ${ }^{(25,51)}$ The experimental PD ligature model creates more aggressive bone loss than LPS injections. ${ }^{(25)}$ This aggressive bone loss may have resulted in the one osteonecrotic lesion in vehicle-treated animals. Importantly, necrotic bone exposure owing to infection or mechanical trauma in the jaws of patients not receiving BPs has been reported. ${ }^{(57-59)}$ However, correlation with the severity of dental disease was not assessed.

BRONJ is a clinically defined disease described as exposed bone for more than 8 weeks. However, we observed bone exposure only in $4(21 \%)$ of the ZA-treated animals, whereas we observed radiographic and histologic osteonecrosis in $47 \%$ of the same animals. This would suggest that bone necrosis precedes mucosal retraction and bone exposure. Indeed, sequestrum formation in patients receiving BPs without signs of clinically exposed bone have been reported. ${ }^{(60)}$

Based on our findings and other published data, we propose a model of BRONJ pathophysiology (Fig. 10) where the combination of robust alveolar bone resorption by BPs and the presence of aggressive dental disease provide a unique environment for BRONJ development. Figure $10 A$ shows a healthy periodontium. The alveolar bone demonstrates a uniform, well-defined cortical outline and normal trabecular pattern. Dental disease such as PD (Fig. 10B) creates an inflammatory environment (gray-shadowed area), inducing alveolar bone resorption (note the crestal bone loss between $A$ and $B$ ). Bone loss in the presence of inflammation could be essential to remove bone from the area and to allow mounting of an effective immune response. In the absence of dental disease, BPs (Fig. 10C) attenuate osteoclastic activity and increase alveolar bone density. Periodontal disease in the presence of BPs (Fig. 10D) similarly creates periodontal tissue inflammation (depicted by the gray-shadowed area). However, osteoclastic activity is greatly diminished. As a result, alveolar bone cannot be resorbed away from the inflammatory nidus, and therefore, the bone is exposed to an environment rich in bacterial toxins, inflammatory cytokines, or oxidative stress. Such an environment is highly toxic to bone cells and results in osteonecrosis. Thus BRONJ develops.

In our rat model of BRONJ, osteocyte necrosis precedes clinical bone exposure. In humans treated with BP, similar subclinical alveolar bone necrosis may be present around teeth with severe periodontal disease, periapical disease, or deep caries. Extraction of such teeth would injure the surrounding gingival tissues and expose the necrotic bone. Our model suggests that these factors in combination have the potential to precipitate clinically diagnostic BRONJ.

Also, our model emphasizes bone resorption as crucial in BRONJ development. This would predict that other strong osteoclast inhibitors could, similar to BPs, prevent protective bone resorption and thus predispose patients to ONJ. Indeed, we and others have reported ONJ in patients receiving denosumab, an anti-RANKL monoclonal antibody. ${ }^{(61,62)}$ The incidence of ONJ development between ZA and denosumab appears to be comparable. ${ }^{(63)}$ 
It is important to note that in our experiments, animals were treated with high-dose ZA to simulate the 4-mg/60-kg monthly dose administered to cancer patients for bone disease control. ${ }^{(23,24)}$ This BP treatment is associated with a significantly higher risk of BRONJ development than BP interventions used in osteoporotic patients. ${ }^{(1,2)}$ Although a significant number of osteoporotic patients in BP treatment have periodontal disease, only a very small fraction of such patients develop BRONJ.

In conclusion, we have created in the rat a condition that closely mimics BRONJ in patients. Although BRONJ pathophysiology is complex and many factors could influence its prevalence, duration, and severity, our data suggest that dental disease and inhibition of bone resorption by a potent BP are necessary and sufficient for BRONJ development.

\section{Acknowledgments}

This work was supported by NIH/NIDCR DE019465 and UCLA's Jonsson Cancer Center Foundation (JCCF) seed grant. The project described also was supported in part by Award Number P30AG028748 from the National Institute on Aging. The content of this article is solely the responsibility of the authors.

\section{References}

1. Khosla S, Burr D, Cauley J, et al. Bisphosphonate-associated osteonecrosis of the jaw: report of a task force of the American Society for Bone and Mineral Research. J Bone Miner Res. 2007; 22:1479-1491. [PubMed: 17663640]

2. Ruggiero SL, Dodson TB, Assael LA, Landesberg R, Marx RE, Mehrotra B. American Association of Oral and Maxillofacial Surgeons position paper on bisphosphonate-related osteonecrosis of the jaws: 2009 update. J Oral Maxillofac Surg. 2009; 67:S2-12.

3. Marx RE, Sawatari Y, Fortin M, Broumand V. Bisphosphonate-induced exposed bone (osteonecrosis/osteopetrosis) of the jaws: risk factors, recognition, prevention, and treatment. J Oral Maxillofac Surg. 2005; 63:1567-1575. [PubMed: 16243172]

4. Marx RE. A new concept in the treatment of osteoradionecrosis. J Oral Maxillofac Surg. 1983; 41:351-357. [PubMed: 6574217]

5. Mehrotra B, Ruggiero S. Bisphosphonate complications including osteonecrosis of the jaw. Hematology, Am Soc Hematol Educ Program. 2006; 356-360:515.

6. Bamias A, Kastritis E, Bamia C, et al. Osteonecrosis of the jaw in cancer after treatment with bisphosphonates: incidence and risk factors. J Clin Oncol. 2005; 23:8580-8587. [PubMed: 16314620]

7. Ruggiero SL, Mehrotra B, Rosenberg TJ, Engroff SL. Osteonecrosis of the jaws associated with the use of bisphosphonates: a review of 63 cases. J Oral Maxillofac Surg. 2004; 62:527-534. [PubMed: 15122554]

8. Tarassoff P, Csermak K. Avascular necrosis of the jaws: risk factors in metastatic cancer patients. $\mathrm{J}$ Oral Maxillofac Surg. 2003; 61:1238-1239. [PubMed: 14586868]

9. Bone HG, Hosking D, Devogelaer JP, et al. Ten years' experience with alendronate for osteoporosis in postmenopausal women. N Engl J Med. 2004; 350:1189-1199. [PubMed: 15028823]

10. Zervas K, Verrou E, Teleioudis Z, et al. Incidence, risk factors and management of osteonecrosis of the jaw in patients with multiple myeloma: a single-centre experience in 303 patients. $\mathrm{Br} \mathrm{J}$ Haematol. 2006; 134:620-623. [PubMed: 16889620]

11. Durie BG, Katz M, Crowley J. Osteonecrosis of the jaw and bisphosphonates. N Engl J Med. 2005; 353:99-102. discussion 99-102. [PubMed: 16000365]

12. Dimopoulos MA, Kastritis E, Anagnostopoulos A, et al. Osteonecrosis of the jaw in patients with multiple myeloma treated with bisphosphonates: evidence of increased risk after treatment with zoledronic acid. Haematologica. 2006; 91:968-971. [PubMed: 16757414]

13. Hoff AO, Toth BB, Altundag K, et al. Frequency and risk factors associated with osteonecrosis of the jaw in cancer patients treated with intravenous bisphosphonates. J Bone Miner Res. 2008; 23:826-836. [PubMed: 18558816] 
14. Walter C, Al-Nawas B, Grotz KA, et al. Prevalence and Risk Factors of BisphosphonateAssociated Osteonecrosis of the Jaw in Prostate Cancer Patients with Advanced Disease Treated with Zoledronate. Eur Urol. 2008

15. Boonyapakorn T, Schirmer I, Reichart PA, Sturm I, Massenkeil G. Bisphosphonate-induced osteonecrosis of the jaws: prospective study of 80 patients with multiple myeloma and other malignancies. Oral Oncol. 2008; 44:857-869. [PubMed: 18282788]

16. Mavrokokki T, Cheng A, Stein B, Goss A. Nature and frequency of bisphosphonate-associated osteonecrosis of the jaws in Australia. J Oral Maxillofac Surg. 2007; 65:415-423. [PubMed: 17307586]

17. Lo JC, O'Ryan FS, Gordon NP, et al. Prevalence of osteonecrosis of the jaw in patients with oral bisphosphonate exposure. J Oral Maxillofac Surg. 2010; 68:243-253. [PubMed: 19772941]

18. Ficarra G, Beninati F, Rubino I, et al. Osteonecrosis of the jaws in periodontal patients with a history of bisphosphonates treatment. J Clin Periodontol. 2005; 32:1123-1128. [PubMed: 16212571]

19. Bilezikian JP. Osteonecrosis of the jaw-do bisphosphonates pose a risk? N Engl J Med. 2006; 355:2278-2281. [PubMed: 17135582]

20. Marx, R. Oral \& Intravenous Bisphosphonate-Induced Osteonecrosis of the Jaws. Quintessence Publishing Co.; Chicago: 2007.

21. Dimopoulos MA, Kastritis E, Bamia C, et al. Reduction of osteonecrosis of the jaw (ONJ) after implementation of preventive measures in patients with multiple myeloma treated with zoledronic acid. Ann Oncol. 2009; 20:117-120. [PubMed: 18689864]

22. Ripamonti CI, Maniezzo M, Campa T, et al. Decreased occurrence of osteonecrosis of the jaw after implementation of dental preventive measures in solid tumour patients with bone metastases treated with bisphosphonates. The experience of the National Cancer Institute of Milan. Ann Oncol. 2009; 20:137-145. [PubMed: 18647964]

23. Kyle RA, Yee GC, Somerfield MR, et al. American Society of Clinical Oncology 2007 clinical practice guideline update on the role of bisphosphonates in multiple myeloma. J Clin Oncol. 2007; 25:2464-2472. [PubMed: 17515569]

24. Quinn R. Comparing rat's to human's age: how old is my rat in people years? Nutrition. 2005; 21:775-777. [PubMed: 15925305]

25. Rogers JE, Li F, Coatney DD, et al. Actinobacillus actinomycetemcomitans lipopolysaccharidemediated experimental bone loss model for aggressive periodontitis. J Periodontol. 2007; 78:550558. [PubMed: 17335380]

26. Bianchi SD, Scoletta M, Cassione FB, Migliaretti G, Mozzati M. Computerized tomographic findings in bisphosphonate-associated osteonecrosis of the jaw in patients with cancer. Oral Surg Oral Med Oral Pathol Oral Radiol Endod. 2007; 104:249-258. [PubMed: 17560140]

27. Bedogni A, Blandamura S, Lokmic Z, et al. Bisphosphonate-associated jawbone osteonecrosis: a correlation between imaging techniques and histopathology. Oral Surg Oral Med Oral Pathol Oral Radiol Endod. 2008; 105:358-364. [PubMed: 18280968]

28. Bi Y, Gao Y, Ehirchiou D, et al. Bisphosphonates cause osteonecrosis of the jaw-like disease in mice. Am J Pathol. 2010; 177:280-290. [PubMed: 20472893]

29. Coleman RE, Major P, Lipton A, et al. Predictive value of bone resorption and formation markers in cancer patients with bone metastases receiving the bisphosphonate zoledronic acid. J Clin Oncol. 2005; 23:4925-4935. [PubMed: 15983391]

30. Stewart AF. Clinical practice. Hypercalcemia associated with cancer. N Engl J Med. 2005; 352:373-379. [PubMed: 15673803]

31. Duivenvoorden WC, Vukmirovic-Popovic S, Kalina M, Seidlitz E, Singh G. Effect of zoledronic acid on the doxycycline-induced decrease in tumour burden in a bone metastasis model of human breast cancer. Br J Cancer. 2007; 96:1526-1531. [PubMed: 17437017]

32. Diel IJ, Body JJ, Lichinitser MR, et al. Improved quality of life after long-term treatment with the bisphosphonate ibandronate in patients with metastatic bone disease due to breast cancer. Eur J Cancer. 2004; 40:1704-1712. [PubMed: 15251160]

33. Lin JT, Lane JM. Osteoporosis: a review. Clin Orthop Relat Res. 2004; 425:126-134. [PubMed: 15292797] 
34. Russell RG, Watts NB, Ebetino FH, Rogers MJ. Mechanisms of action of bisphosphonates: similarities and differences and their potential influence on clinical efficacy. Osteoporos Int. 2008; 19:733-759. [PubMed: 18214569]

35. Chung WO, Dommisch H, Yin L, Dale BA. Expression of defensins in gingiva and their role in periodontal health and disease. Curr Pharm Des. 2007; 13:3073-3083. [PubMed: 17979750]

36. Taubman MA, Nash DA. The scientific and public-health imperative for a vaccine against dental caries. Nat Rev Immunol. 2006; 6:555-563. [PubMed: 16729013]

37. Slade GD, Beck JD. Plausibility of periodontal disease estimates from NHANES III. J Public Health Dent. 1999; 59:67-72. [PubMed: 10965470]

38. Allen MR, Burr DB. The pathogenesis of bisphosphonate-related osteonecrosis of the jaw: so many hypotheses, so few data. J Oral Maxillofac Surg. 2009; 67(5 Suppl):61-70. [PubMed: 19371816]

39. Allen MR, Burr DB. Mandible matrix necrosis in beagle dogs after 3 years of daily oral bisphosphonate treatment. J Oral Maxillofac Surg. 2008; 66:987-994. [PubMed: 18423290]

40. Burr DB, Allen MR. Mandibular necrosis in beagle dogs treated with bisphosphonates. Orthod Craniofac Res. 2009; 12:221-228. [PubMed: 19627524]

41. Sonis ST, Watkins BA, Lyng GD, Lerman MA, Anderson KC. Bony changes in the jaws of rats treated with zoledronic acid and dexamethasone before dental extractions mimic bisphosphonaterelated osteonecrosis in cancer patients. Oral Oncol. 2009; 45:164-172. [PubMed: 18715819]

42. Kikuiri T, Kim I, Yamaza T, et al. Cell-based immunotherapy with mesenchymal stem cells cures bisphosphonate-related osteonecrosis of the jaw-like disease in mice. J Bone Miner Res. 2010; 25:1668-1679. [PubMed: 20200952]

43. Hokugo A, Christensen R, Chung EM, et al. Increased prevalence of bisphosphonate-related osteonecrosis of the jaw with vitamin D deficiency in rats. J Bone Miner Res. 2010; 25:13371349. [PubMed: 20200938]

44. Gotcher JE, Jee WS. The progress of the periodontal syndrome in the rice cat. II. The effects of a diphosphonate on the periodontium. J Periodontal Res. 1981; 16:441-455. [PubMed: 6459441]

45. Gotcher JE, Jee WS. The progress of the periodontal syndrome in the rice rat. I. Morphometric and autoradiographic studies. J Periodontal Res. 1981; 16:275-291. [PubMed: 6458677]

46. Ferreira RI, de Almeida SM, Boscolo FN, Santos AO, Camargo EE. Bone scintigraphy as an adjunct for the diagnosis of oral diseases. J Dent Educ. 2002; 66:1381-1387. [PubMed: 12521065]

47. Arias JA, Pardo C, Olmos A, Cuadrado ML, Ruibal A. Dental diseases and radionuclide imaging of the jaws. Nucl Med Commun. 2004; 25:305-310. [PubMed: 15094451]

48. Lyons KP, Jensen JL. Dental lesions causing abnormalities on skeletal scintigraphy. Clin Nucl Med. 1979; 4:509-512. [PubMed: 527288]

49. Strittmatter EJ, Keller DL, LaBounty GL, Lewis DM, Graham GD. The relationship between radionuclide bone scans and dental examinations. Oral Surg Oral Med Oral Pathol. 1989; 68:576581. [PubMed: 2812712]

50. Tow DE, Garcia DA, Jansons D, Sullivan TM, Niederman R. Bone scan in dental diseases. J Nucl Med. 1978; 19:845-847. [PubMed: 660289]

51. de Almeida JM, Theodoro LH, Bosco AF, Nagata MJ, Oshiiwa M, Garcia VG. Influence of photodynamic therapy on the development of ligature-induced periodontitis in rats. J Periodontol. 2007; 78:566-575. [PubMed: 17335382]

52. Badran Z, Kraehenmann MA, Guicheux J, Soueidan A. Bisphosphonates in periodontal treatment: a review. Oral Health Prev Dent. 2009; 7:3-12. [PubMed: 19408809]

53. Allen MR, Follet H, Khurana M, Sato M, Burr DB. Antiremodeling agents influence osteoblast activity differently in modeling and remodeling sites of canine rib. Calcif Tissue Int. 2006; 79:255-261. [PubMed: 17033724]

54. Feher A, Koivunemi A, Koivunemi M, et al. Bisphosphonates do not inhibit periosteal bone formation in estrogen deficient animals and allow enhanced bone modeling in response to mechanical loading. Bone. 46:203-207. [PubMed: 19857619]

55. Kashii M, Hashimoto J, Nakano T, Umakoshi Y, Yoshikawa H. Alendronate treatment promotes bone formation with a less anisotropic microstructure during intramembranous ossification in rats. J Bone Miner Metab. 2008; 26:24-33. [PubMed: 18095060] 
56. Ekuni D, Yamamoto T, Yamanaka R, Tachibana K, Watanabe T. Proteases augment the effects of lipopolysaccharide in rat gingiva. J Periodontal Res. 2003; 38:591-596. [PubMed: 14632922]

57. Meer S, Coleman H, Altini M, Alexander T. Mandibular osteomyelitis and tooth exfoliation following zoster-CMV co-infection. Oral Surg Oral Med Oral Pathol Oral Radiol Endod. 2006; 101:70-75. [PubMed: 16360610]

58. Farah CS, Savage NW. Oral ulceration with bone sequestration. Aust Dent J. 2003; 48:61-64. [PubMed: 14640160]

59. Schwartz HC. Osteonecrosis of the jaws: a complication of cancer chemotherapy. Head Neck Surg. 1982; 4:251-253. [PubMed: 6896046]

60. Junquera L, Gallego L. Nonexposed bisphosphonate-related osteonecrosis of the jaws: another clinical variant? J Oral Maxillofac Surg. 2008; 66:1516-1517. [PubMed: 18571043]

61. Aghaloo TL, Felsenfeld AL, Tetradis S. Osteonecrosis of the jaw in a patient on Denosumab. J Oral Maxillofac Surg. 68:959-963. [PubMed: 20149510]

62. Taylor KH, Middlefell LS, Mizen KD. Osteonecrosis of the jaws induced by anti-RANK ligand therapy. Br J Oral Maxillofac Surg. 2010; 48:221-223. [PubMed: 19836866]

63. Kyrgidis A, Toulis KA. Denosumab-related osteonecrosis of the jaws. Osteoporosis Int. 2011; 22:369-370. 

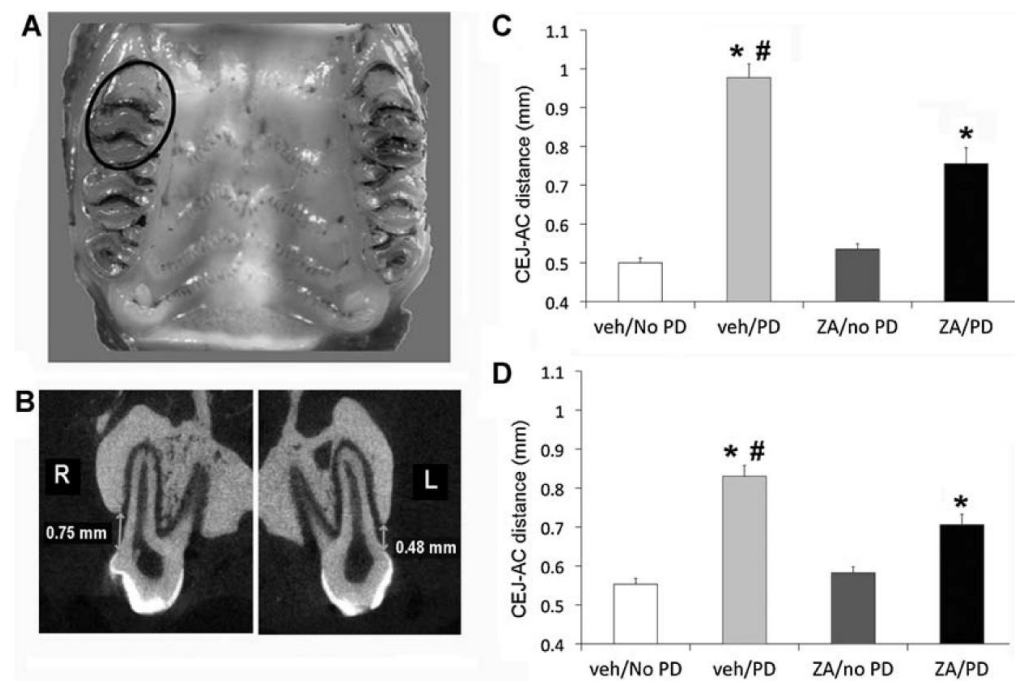

Fig. 1.

(A) Diagram of in vivo periodontal disease model. A sterile ligature was placed subgingivally around the maxillary first molar unilaterally (blue circle) to induce periodontal disease in vehicle- or ZA-treated animals. $(B)$ To quantify ligature-induced alveolar bone loss, the distance between the cementoenamel junction (CEJ) and alveolar crest (AC) in ligated $(R)$ and nonligated $(L)$ sites was measured. Longer distance denotes bone loss. (C) CEJ-AC distance at the distal buccal root of the first molar (D1). (D) CEJ-AC distance at the mesial buccal root of the second molar (M2). *Statistically significantly greater than the nonligated site. ${ }^{\#}$ Statistically significantly greater than ZA-treated animals, $p<.01$. 

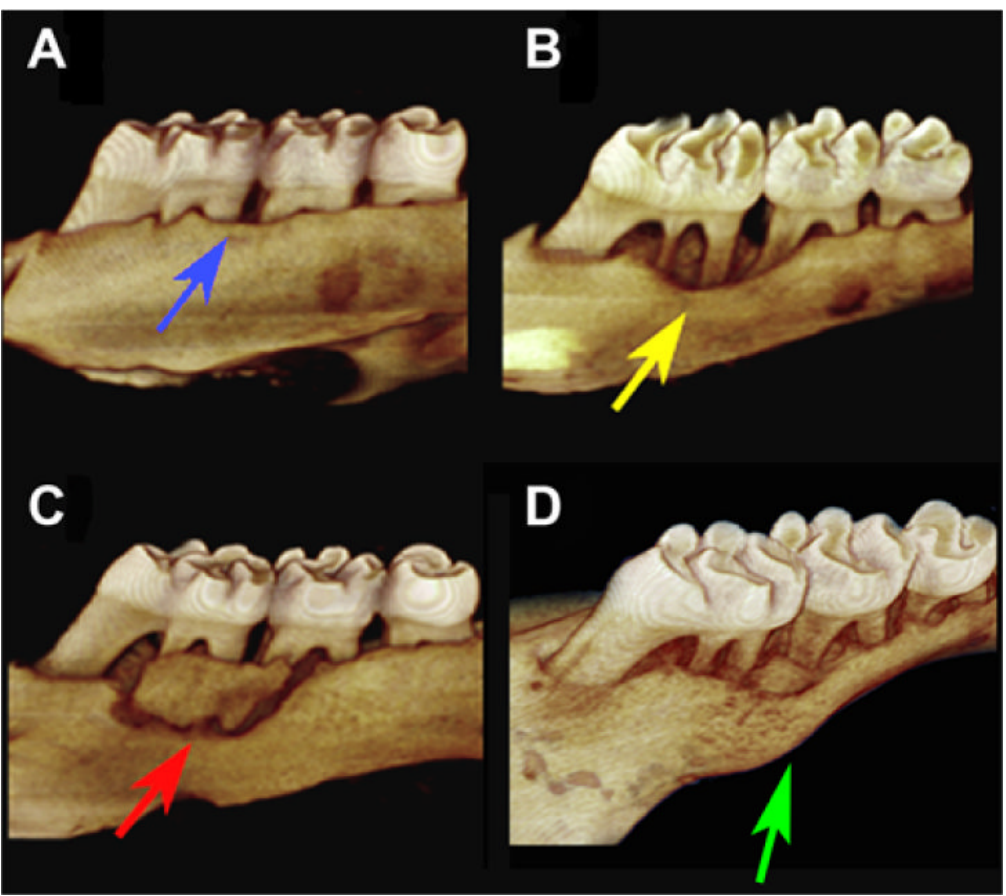

Fig. 2.

3D $\mu \mathrm{CT}$ reconstructed images of the rat maxilla. $(A)$ Unligated site in vehicle-treated rat. $(B)$ Ligature-induced periodontal disease in vehicle-treated rat. $(C, D)$ Ligature-induced periodontal disease in ZA-treated rat. Blue arrow in panel $A$ points to normal alveolar bone in the interproximal area of the distal root of the first molar and mesial root of the second molar. The yellow arrow in panel $B$ points to periodontal bone loss in vehicle-treated animals at the area of the ligature. In ZA-treated animals, red arrow points to a sequestrum formation $(C)$, and green arrow points to extensive periosteal new bone formation $(D)$ in the area of the ligature. 


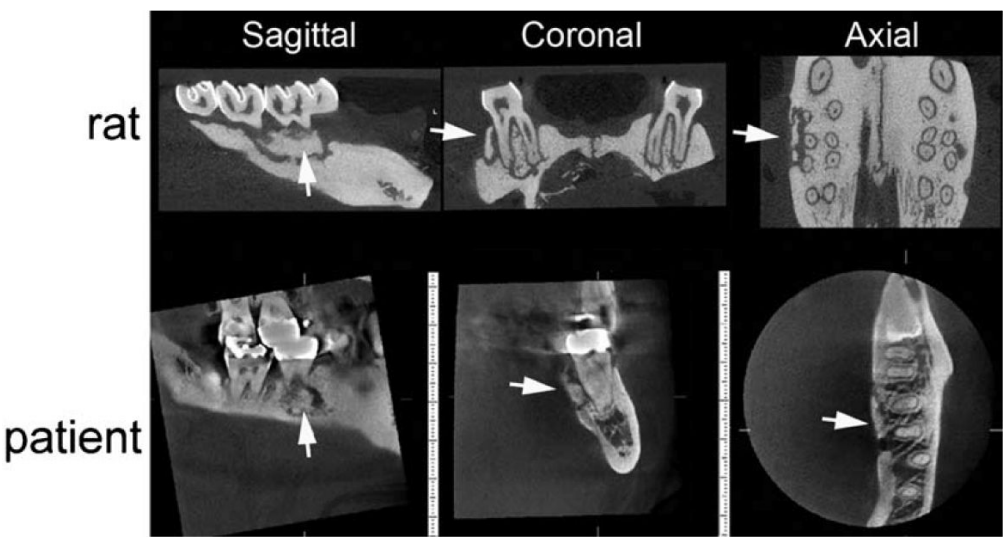

Fig. 3.

Similarity of rat and human radiographic findings in BRONJ. Sagittal, coronal, and axial CT slices from a rat and a patient with BRONJ demonstrate a bony sequestrum in the alveolar bone (arrows). 


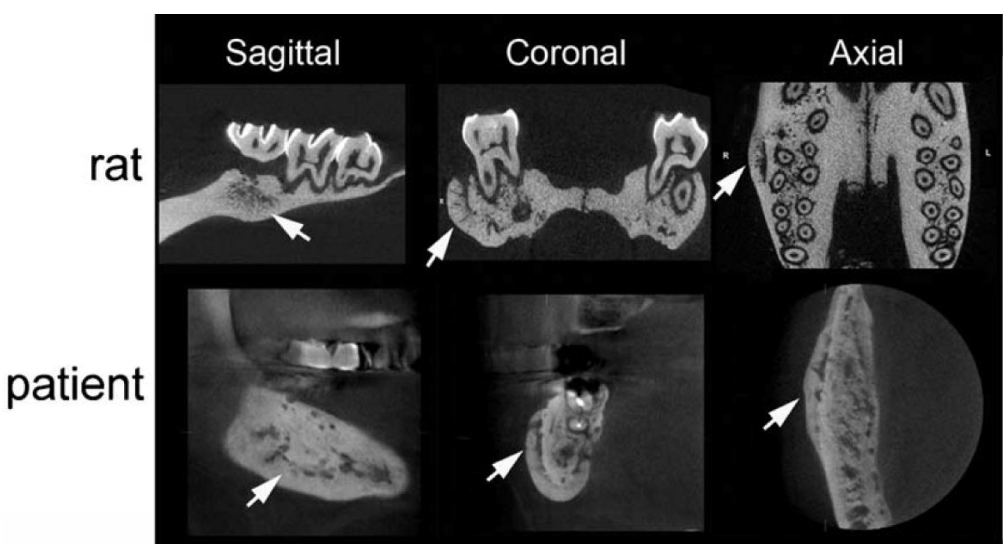

Fig. 4.

Similarity of rat and human radiographic findings in BRONJ. Sagittal, coronal, and axial CT slices from a rat and a patient with BRONJ demonstrate significant periosteal bone reaction and expansion of the alveolar ridge dimensions (arrows). 

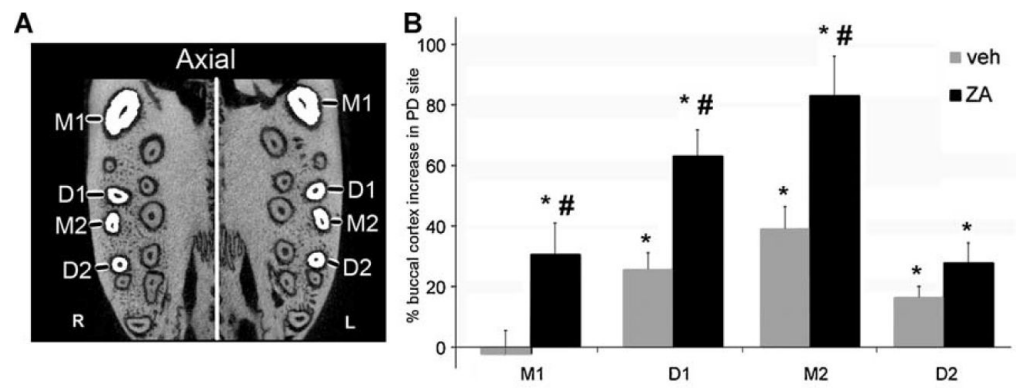

C
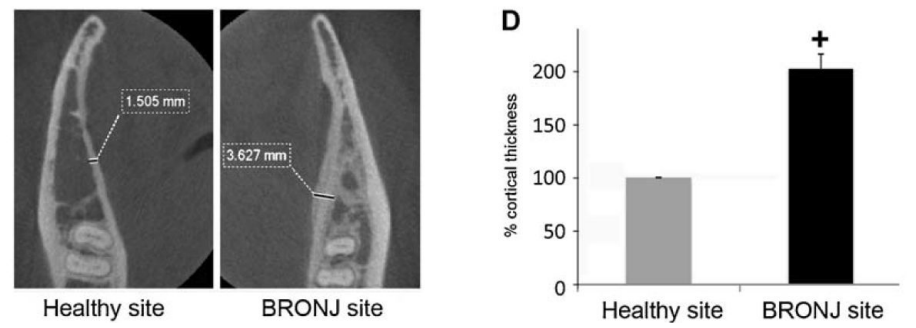

Fig. 5.

Comparison of periosteal bone formation in BRONJ and healthy sites in the rat and patient. To quantify buccal bone thickness in vehicle- versus ZA-treated rats, the buccal width of the alveolar bone was measured at the mesial buccal and distal buccal roots of the first (M1 and D1) and second (M2 and D2) molars in the ligated and nonligated sites on axial $\mu \mathrm{CT}$ slices $(A)$. The measurements on the PD site were calculated as a percentage of the same measurements on the healthy site, and the percent increase was determined $(B)$. To evaluate bone width in BRONJ-involved versus noninvolved sites, cortical bone thickness was measured at the BRONJ site and the same alveolar bone area of the noninvolved site on CBCT images of patients $(C)$. Cortical thickness was expressed as percent thickness of the noninvolved site $(D)$. ${ }^{*}$ Statistically significantly different from the non-PD site, $p<$.

01. "Statistically significantly different from the vehicle- treated group, $p<.01 .{ }^{+}$tatistically significantly different from the noninvolved site, $p<.01$. 

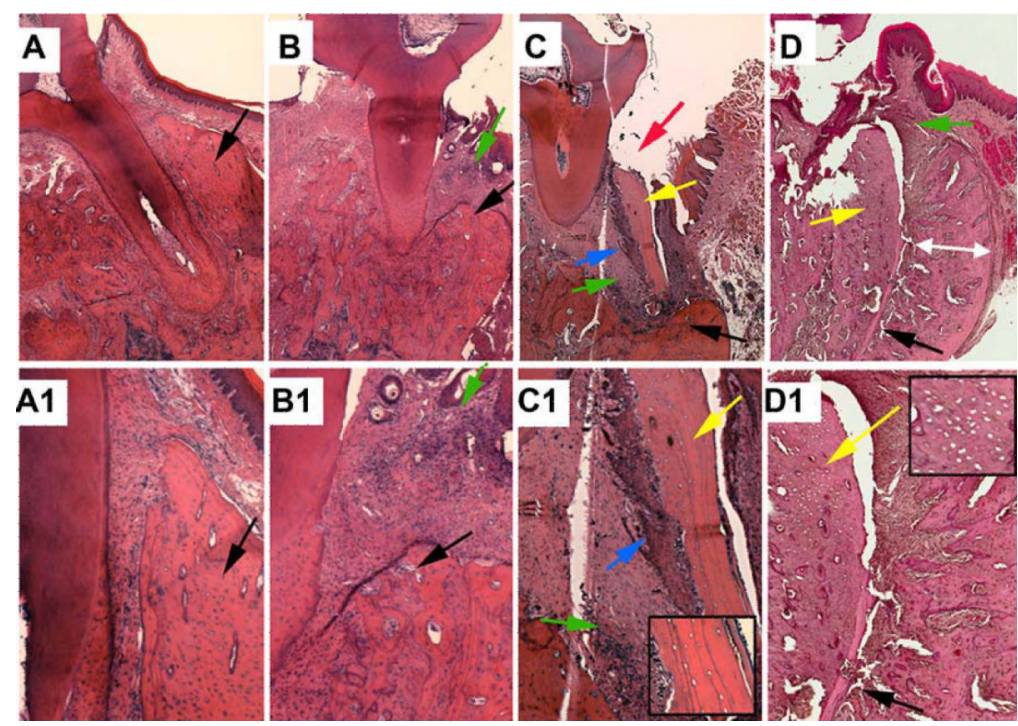

Fig. 6.

Histologic examination of experimentally induced BRONJ. $(A, A I)$ Nonligated site in vehicle-treated animal. $(B, B 1)$ Ligature-induced periodontal disease in untreated rat. $(C$, $C 1, D, D 1)$ Ligature-induced periodontal disease in ZA-treated rat. $(A-D: \times 10$

magnification; $A 1-D 1$ demonstrate a magnified area of $A-D$.) Black arrows point to viable bone, yellow arrows to necrotic bone, green arrows to inflammatory infiltrate, blue arrows to epithelial rimming of necrotic bone, red arrow to interruption of epithelial continuity, and double white arrow to periosteal bone formation. (Inset: $C 1, D 1)$ Extensive osteocyte loss with confluent empty lacunae $(\times 40)$. 

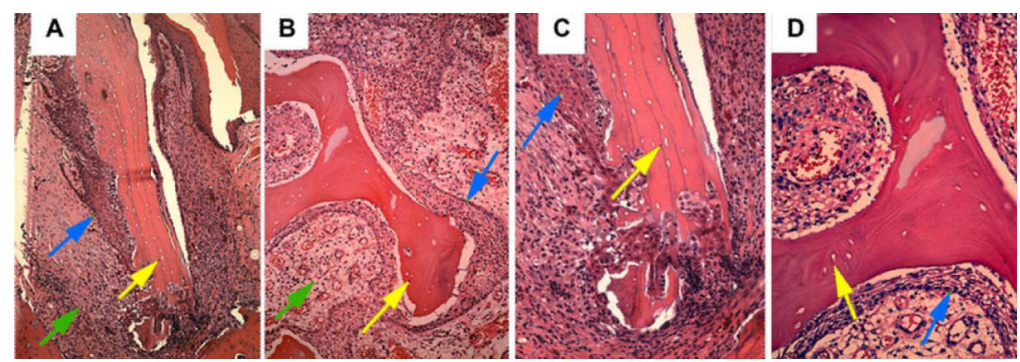

Fig. 7.

Rat and human histologic findings in BRONJ. Sections through the jaw of rat $(A: \times 20 ; C$ : $\times 40)$ and patient $(B: \times 20 ; D: \times 40)$ show osteonecrosis (yellow arrows) with squamous epithelium rimming necrotic bone (blue arrows) and inflammation (green arrows). 

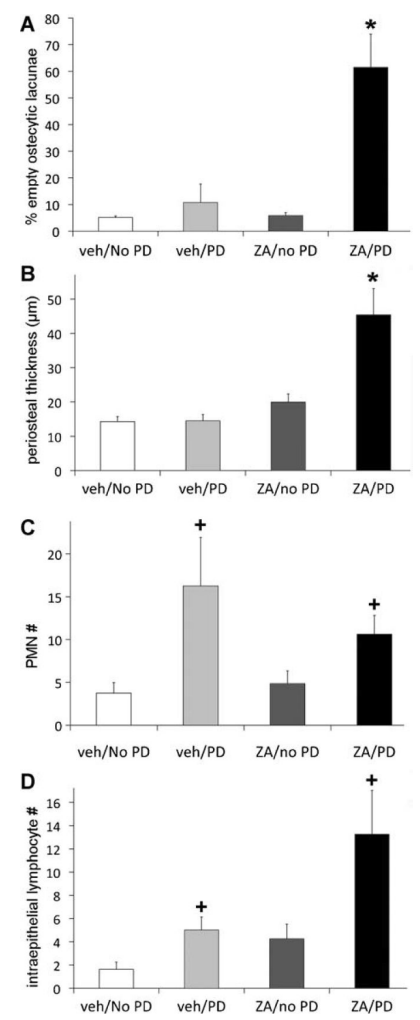

Fig. 8 .

$(A)$ Number of osteocytic lacunae at the buccal alveolus was measured, and empty lacunae were expressed as a percent of total. $(B)$ Thickness of periosteum at the buccal alveolus was determined. (C) Number of polymorphonuclear neutrophils and $(D)$ intraepithelial lymphocytes was quantified. *Statistically significantly greater than the nonligated site of ZA-treated animals and the nonligated or ligated site of vehicle-treated animals, $p<$. 01 . ${ }^{+}$Statistically significantly greater than the nonligated site, $p<.05$. 

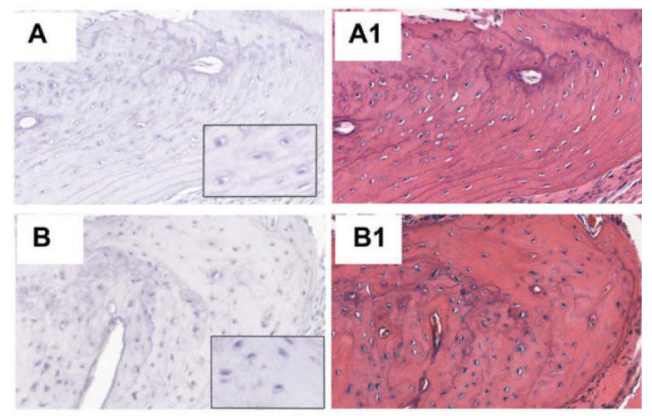

\section{B1}
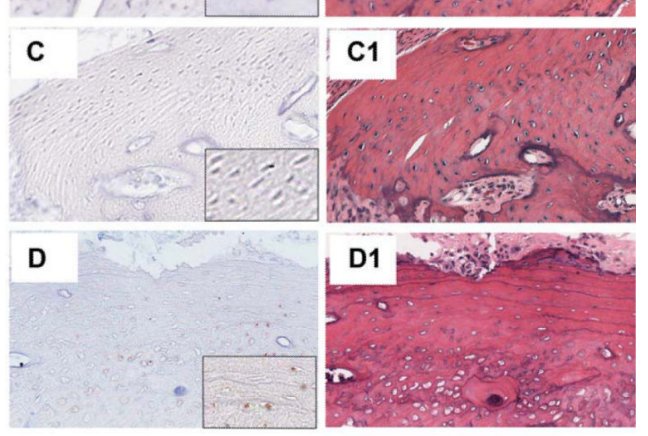

E

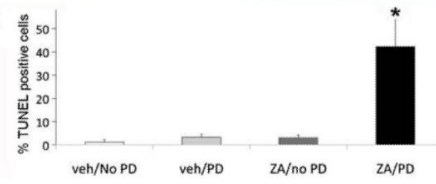

Fig. 9.

$\times 20$ TUNEL-stained $(A-D)$ and H\&E-stained $(A 1-D 1)$ adjacent sections from nonligated $(A, A 1)$ and ligated $(B, B 1)$ sites of a vehicle-treated animal or nonligated $(C, C 1)$ and ligated $(D, D 1)$ sites of a ZA-treated animal. Several TUNEL ${ }^{+}$osteocytes are present in the ZA-treated animal adjacent to an osteonecrotic area $(D 1)$. No apoptotic figures are seen in $(A-C)$. (Insets) $\mathrm{TUNEL}^{-}$and $\mathrm{TUNEL}^{+}$cells in detail $(\times 40)$. $(E)$ Total osteocytes were counted, and percent of TUNEL ${ }^{+}$osteocytes was determined. *Statistically significantly greater than the nonligated site of ZA-treated animals and the nonligated or ligated sites of vehicle-treated animals, $p<.01$. 

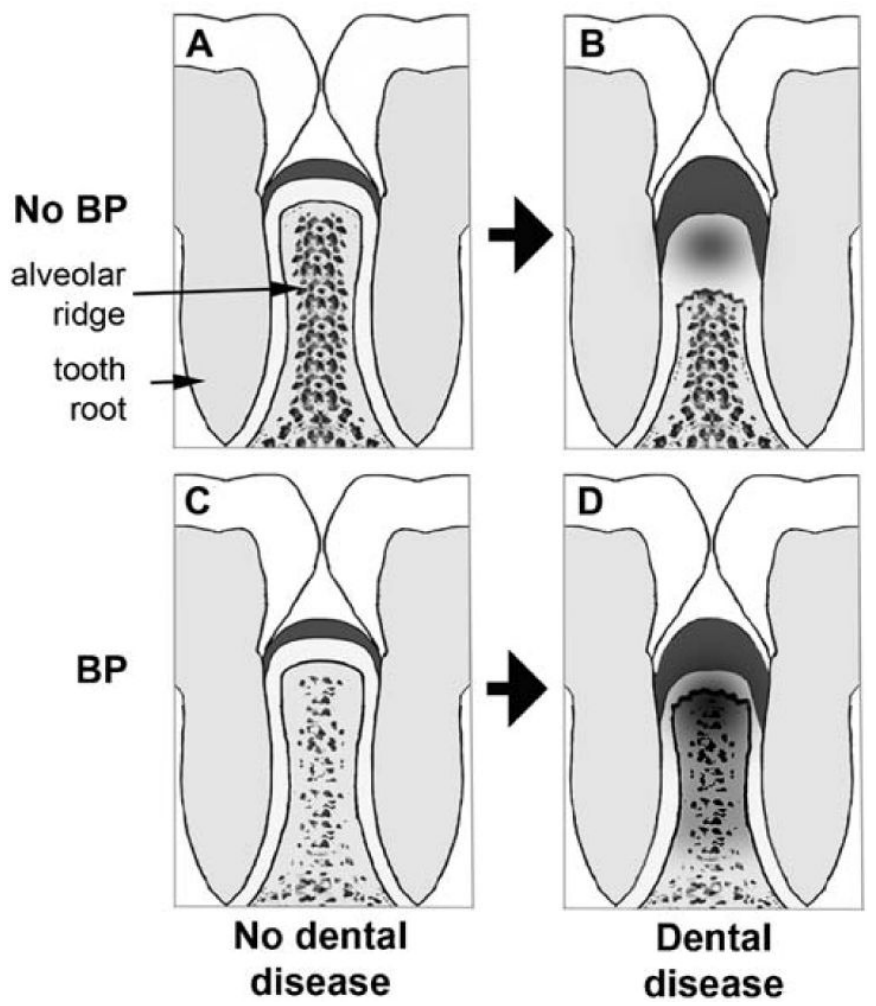

Fig. 10.

Model of BRONJ pathophysiology. Response of alveolar bone to health $(A, C)$ or dental disease $(B, D)$ in the absence $(A, B)$ or presence $(C, D)$ of BP treatment. BRONJ, depicted by the darker color in bone in panel $D$ requires the presence of both BP and dental disease. 


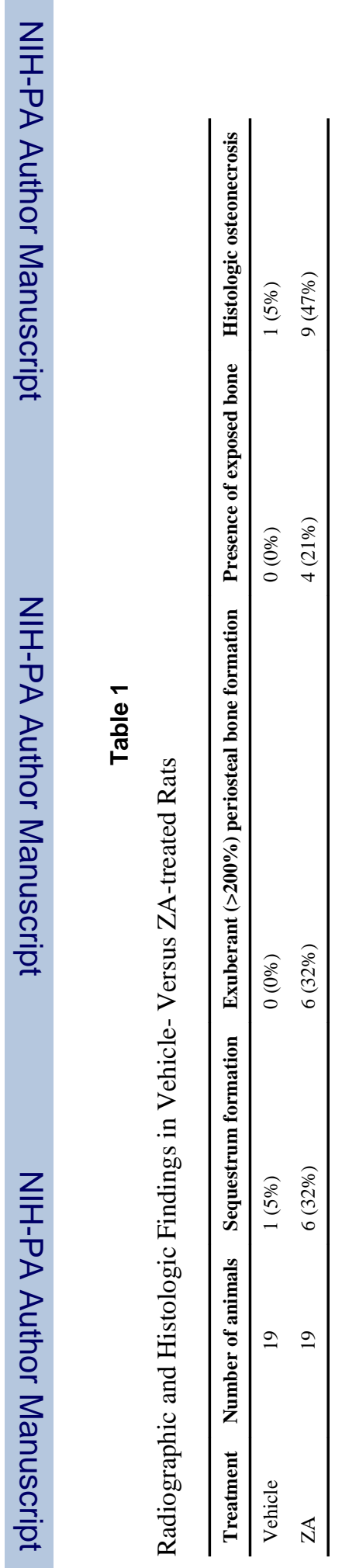

